\title{
Optical Coherence Tomography Findings in Patients with Myotonic Dystrophy Type 1
}

\author{
(D) Onur Akan1, (D) Burak Erden² \\ 1 University of Health Sciences Turkey, Prof. Dr. Cemil Taşçığlu City Hospital, Clinic of Neurology, Istanbul, Turkey \\ 2University of Health Sciences Turkey, Prof. Dr. Cemil Taşçıŏlu City Hospital, Clinic of Eye Diseases, istanbul, Turkey
}

\section{Abstract}

Objective: Myotonic dystrophy type 1 (DM1) is characterized by myotonia and progressive muscle weakness caused by the cytosine-thymineguanine trinucleotide repeat and is the most common muscular dystrophy in adults. Although various ocular abnormalities, particularly cataracts, are well known in patients with DM1, few studies and case reports are available on retinal involvement, such as retinal degeneration, the epiretinal membrane (ERM), and macular degeneration. In this study, we investigated the retinal involvement findings using optical coherence tomography (OCT) in patients who were followed up in the neuromuscular outpatient clinic with a diagnosis of DM1.

Methods: In this prospective cross-sectional study, 20 patients with genetically confirmed DM1 aged 18-65 years were included. The control group was formed from healthy individuals of a similar age and gender distribution to the patient group. Patients with diabetes mellitus, diabetic retinopathy, glaucoma, and previous cataract surgery were excluded from the study. Muscular impairment rating (MIR) scales were evaluated according to the distribution findings of myotonia and muscle weakness on a neurological examination. Patients were evaluated with OCT to examine macular and optic nerve morphology after ophthalmologic and fundus examinations were performed by a retinal specialist.

Results: The mean central macular thickness (CMT) values were $275.52 \pm 40.31$ and $262.76 \pm 9.06$ in the control and study groups, respectively $(p=0.09)$. Subfoveal choroidal thicknesses (SFCT) were significantly higher in the study group (341.8 \pm 95.04 vs. 279.59 $\pm 46.65 ; p=0.002)$. The mean retinal nerve fiber layer did not differ between the groups (103.6 \pm 14 vs. $101.3 \pm 12 ; p=0.12)$. No ERM was detected in any of the DM1 patients. No correlation was found between CMT, SFCT, and the MIR stages $(p<0.05)$.

Conclusion: Visual disturbances associated with DM1 may be caused by cataracts as well as retinal disorders. An OCT examination is a beneficial part of the routine ophthalmologic evaluation in DM1 patients.

Keywords: Myotonic dystrophy type 1, OCT, retinopathy, macular degeneration, retina

\section{INTRODUCTION}

Myotonic dystrophy type 1 (DM1) is a very rare disease that can involve multiple systems, such as skeletal muscle, heart, smooth muscle, the eyes, or the endocrine and central nervous systems. DM1 exhibits an autosomal dominant inheritance pattern caused by the cytosine-thymine-guanine trinucleotide repeat $(1,2)$. The disease is characterized by myotonia and progressive muscle weakness, and it is the most common muscular dystrophy in adults $(1,2)$. Although various ocular abnormalities, particularly cataracts, are well known in patients with DM1, few studies and case reports are available on retinal involvement, such as retinal degeneration, the epiretinal membrane (ERM), and macular degeneration. In this study, we investigated the retinal involvement findings using optical coherence tomography (OCT) in patients who were followed up in the neuromuscular outpatient clinic with a diagnosis of DM1. As an OCT examination is a fast and noninvasive instrument to search for posterior segment ocular 
pathologies without pupillary dilatation, it might be a helpful tool to investigate the etiology of the visual impairment in DM1 patients.

\section{METHODS}

In this prospective cross-sectional study, 20 patients with genetically confirmed DM1 aged 18-65 years who were followed up in the Neuromuscular Outpatient Clinic of University of Health Sciences Turkey, Prof. Dr. Cemil Taşcıoğlu City Hospital were included in the study. Patients with diabetes mellitus, diabetic retinopathy (DR), glaucoma, and previous cataract surgery were excluded from the study. A control group was formed from healthy individuals of similar age and gender distribution to the patient group. Demographic and clinical data of the patients, such as age, gender, and disease duration, were noted. The muscle strength examination of the patients involved 11 muscle groups including the bilateral neck flexors. The proximal and distal muscle groups were scored on the medical research council (MRC) (0-5 points) scale by manual muscle testing. Muscular impairment rating (MIR) scales were evaluated according to the distribution findings of myotonia and muscle weakness on the neurological examination (3). Accordingly, the patients were classified into five grades as patients without clinical muscle weakness in grade 1 , clinical myotonia without limb weakness and weakness of the face and neck flexors in grade 2, distal weakness in grade 3 , mild to moderate (MRC score $<5)$ proximal weakness in grade 4 , and severe (MRC score $<3$ ) proximal weakness in grade 5 .

Patients were evaluated by OCT to examine the macular and optic nerve morphology after ophthalmologic and fundus examinations were performed by a retinal specialist. Macular and retinal nerve thickness measurements were made in both eyes. Choroidal thickness was evaluated using the enhanced depth imaging mode of

spektral domain (SD)-OCT (Spectralis HRA + OCT; Heidelberg Engineering Inc., Heidelberg, Germany). Subfoveal choroidal thickness (SFCT) was measured between the hyperreflective retinal pigment epithelium-Bruch membrane complex and the hyperreflective scleral/choroidal junction (Figure 1). The presence of the ERM and similar pathologies were investigated. The ophthalmological examination findings, biomicroscopic findings, OCT findings [central macular thickness, retinal nerve fiber thickness (RNFL)], and OCT comments were recorded. The characteristics of the DM1 patients and control groups were compared.

\section{Statistical Analysis}

Statistical analyses were performed using SPSS 22.0 statistical software (SPSS Inc., Chicago, IL, USA). The data were determined to be normally distributed by the Kolmogorov-Smirnov test. Descriptive summary statistics are expressed as means (range and SD) for continuous variables and as frequencies and percentages for categorical variables. The relationships between the categorical data were evaluated with the chi-square test. To compare two independent groups, the Student's t-test was used for normally distributed data, whereas the Mann-Whitney test was used for the not normally distributed data. Spearman's correlation test was used to evaluate the relationship between disease duration and the MIR scale. A $p$ value $<0.05$ was considered significant.

Ethics committee approval was obtained for the study from University of Health Sciences Turkey, Prof. Dr. Cemil Taşçıoğlu City Hospital Location Ethics Committee (30.06.2020-277). It was made following the principles of the Declaration of Helsinki.

\section{RESULTS}

Twenty patients (10 males and 10 females; mean age $36.4 \pm 14.5$; range 18-65 years) were included in the study group. The mean symptom duration of the patients was 10.8 years. According to the MIR scale, $10 \%$ of the patients were grade $1,25 \%$ were grade 2 , $25 \%$ were grade $3,20 \%$ were grade 4 , and $20 \%$ were grade 5 .

A choroidal neovascular membrane (CNVM) was found in one eye of one patient with retinal pigmentary changes in the fellow eye (Figure 2, 3). In another patient, paracentral acute middle maculopathy (PAMM) secondary to a possible transient arterial obstruction was detected on the OCT scan. A posterior subcapsular cataract was detected in $10 \%$ of the patients $(n=2)$. No evidence of ERMs was detected in any of the DM1 patients.

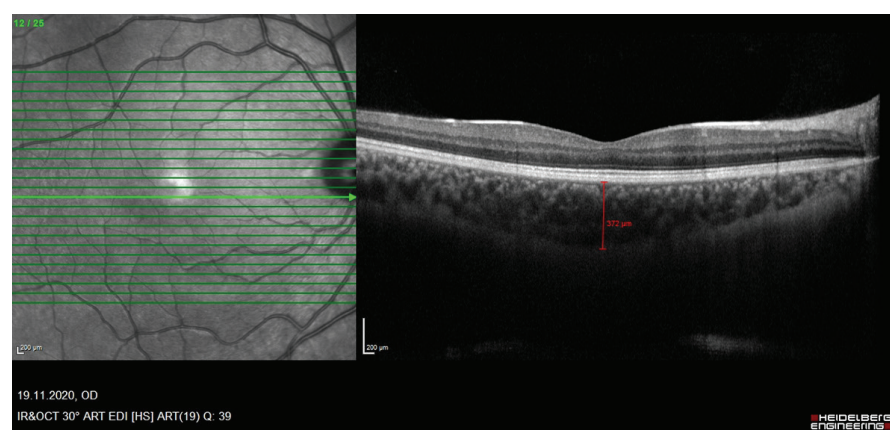

Figure 1. SFCT was measured manually by an experienced retinal physician using an integrated caliper system SFCT: Subfoveal choroidal thicknesses 
When OCT findings were examined in the study patients (40 eyes of 20 patients), the mean central macula thickness (CMT) was $275.52 \pm 40.31 \mu \mathrm{m}$. The mean CMT in the control group was $262.76 \pm 9.06 \mu \mathrm{m}(p=0.09)$. The mean SFCT differed between the

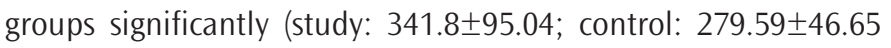
$\mu \mathrm{m} ; \mathrm{p}=0.002)$. The mean RNFL values were 103.6 \pm 14 and $101.3 \pm 12$ $\mu \mathrm{m}$ in the study and control groups, respectively $(p=0.12)$.

No significant correlation was found between the MIR stages and the CMT, RNFL, or SFCT values of the study group ( $p>0.05)$.

The demographic, clinical, and OCT findings of the study and control groups are shown in Table 1.

\section{DISCUSSION}

In this study, the presence of retinal changes in patients with very rare DM1 disease was investigated via SD-OCT examinations. The central macular thickness, vitreoretinal interface, and SFCT were evaluated and compared to an age and sex-matched control group. Kersten et al. (4) reported a higher incidence of ERMs among DM1 patients. However, we did not detect the presence of ERMs in any of our patients. However, the mean SFCT was significantly higher in the DM1 patients than in the control group.

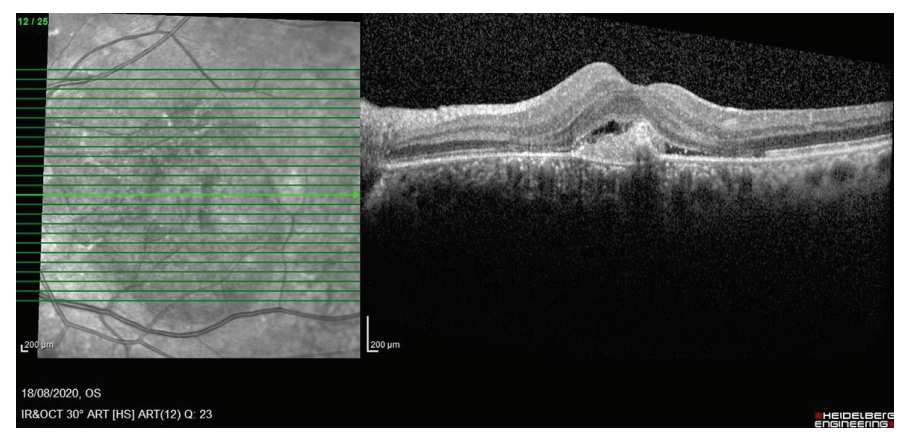

Figure 2. Type 1 CNVM was detected in the left eye of a 59-year-old male patient; the patient was recommended for anti-VEGF therapy CNVM: Choroidal neovascular membrane, VEGF: Vascular endothelial growth factor

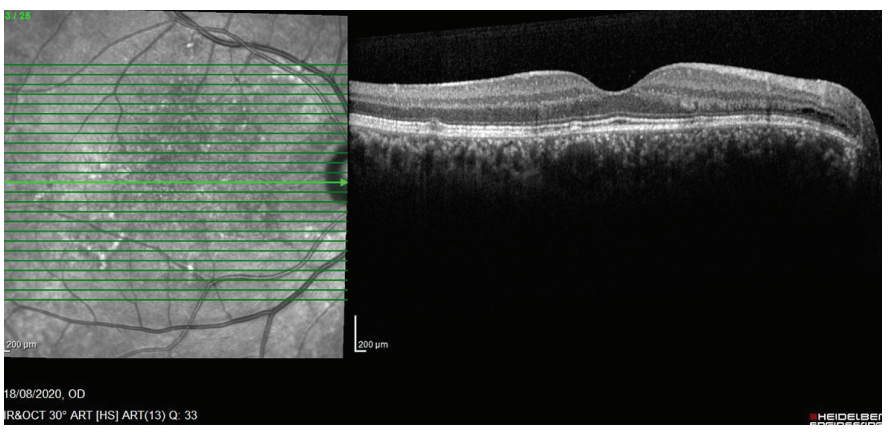

Figure 3. Retinal pigmentary changes were remarkable in the fellow eye of the same patient
To the best of our knowledge, choroidal thickening has not been reported in DM1 patients. The choroid is a very highly vascular tissue that plays an important role in the pathophysiology of many chorioretinal diseases, such as central serous chorioretinopathy (5). Several studies have indicated that choroidal thickness can increase as part of various inflammatory, rheumatologic, metabolic, and systemic diseases $(6,7)$. Metabolic and endocrinologic pathologies are the most reported factors regarding the thickening of the choroid. Kurt et al. (8) reported that the SFCT was higher in inactive Graves disease patients than in a healthy control population. DR and its' effect on choroidal thickness is a wellresearched topic. Choi et al. (9) reported on a large series of DR patients in whom treatment-naïve eyes with proliferative or severe non-proliferative DR had thicker choroids than eyes with mild to moderate non-proliferative DR, indicating a relationship between choroidal thickness and the DR stage (9). Additionally, some studies have reported that choroidal thickening decreases in response to diabetic macular edema treatment, such as intravitreal anti-vascular endothelial growth factor (VEGF) agents (10). Diabetes mellitus, insulin resistance,

Table 1. Demographic, clinical, and OCT findings of the study and control groups

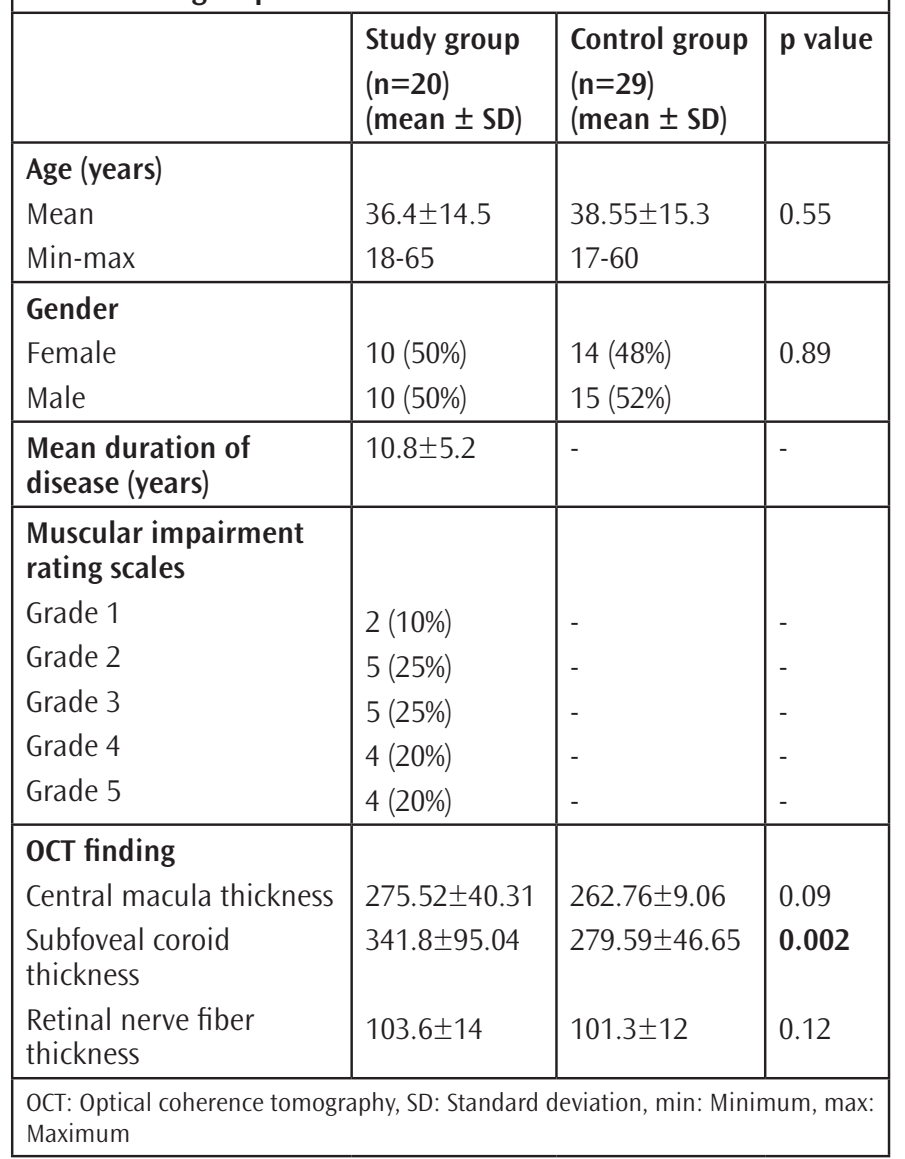


and dyslipidemia are common in patients with DM1 due to endocrine involvement $(1,2,11)$. However, the reason for the significantly higher SFCT in our study group remains unclear. Although we did not include patients manifesting with diabetes mellitus, our study participants might have a prediabetic status affecting the SFCT or the choroidal tissue was thickened in DM1 secondary to other metabolic processes than glycemic pathways.

In addition to muscular dystrophy, many systemic findings, such as endocrinological, gastrointestinal, and cardiac systems, can accompany DM1 (11). The most common ocular involvement finding in DM1 is presenile cataract formation at rates of 90\% (12). In our study group, the presence of cataracts was detected in only $10 \%$ of eyes. The reason for this low cataract rate might be the relatively young age of our study group.

Ptosis, ocular motility disorders, low intraocular pressure, and retinal pigment changes are other ocular abnormalities reported in patients with DM1 (12). Kersten et al. (4) observed signs of high ERM rates in patients with myotonic dystrophy. ERMs are a treatable cause of visual impairment that can lead to reduced best-corrected visual acuity (BCVA) and a reduction in other visual symptoms, such as metamorphosis and micropsy. ERMs are likely to be asymptomatic in DM1 patients because BCVA is affected in stage 2 and later ERM stages. The pathophysiology that causes an increase in ERM in patients with DM1 is unclear. In the general population, ERMs are associated with a variety of ocular abnormalities and systemic diseases, such as intraocular inflammation, retinal vein occlusion, DR, and trauma-causing conditions, including cataract extraction surgery $(4,13-16)$. The reason why we did not detect any ERMs in our DM1 group might be because we included younger patients without diabetes mellitus and excluded pseudophakic eyes.

Kim et al. (17) reported a case with pigmentary retinopathy. In our case series, the CNVM was detected in the left eye and retinal pigmentary changes were observed in the fellow eye of the same patient. Zinkernagel et al. (18) reported a single case of a young DM patient with type 1 CNVM whom they treated with anti-VEGF injections. The presence of type 1 CNVM patient in our cohort was attributed to his older age rather than to DM1.

Posterior subcapsular cataracts were detected in two eyes, but cataract surgery was not planned because of good vision. PAMM was incidentally found in one eye of our patients. This situation was thought to be secondary to a temporary arterial obstruction, as PAMM has not been reported in DM1 patients.

\section{Study Limitations}

Our study had some limitations. The small sample size of our study group limited statistical power but DM1 is a rare disease and our study reflects the findings of a single center. We excluded pseudophakic patients to eliminate the possible effect of cataract surgery on the formation of ERM. Our study group consisted of younger DM1 patients compared to the study groups of previous reports and this may have affected the incidence of vitreomacular and retinal disorders in our group.

\section{CONCLUSION}

OCT is a readily available, fast, and accurate method to identify retinal pathologies, such as ERMs, even in the presence of cataracts. Additionally, the visual disturbances associated with myotonic dystrophy are not only caused by cataracts but by a variety of retinal disorders. We strongly recommend an OCT examination for DM1 patients as a part of the routine ophthalmologic evaluation. Further prospective studies will be conducted in a larger series of older patients.

\section{Ethics}

Ethics Committee Approval: Ethics committee approval was obtained for the study from University of Health Sciences Turkey, Prof. Dr. Cemil Taş̧̧ığlu City Hospital Location Ethics Committee (30.06.2020-277). It was made following the principles of the Declaration of Helsinki.

Informed Consent: Written consent was obtained from the patient for the use of images.

Peer-review: Externally peer-reviewed.

\section{Authorship Contributions}

Surgical and Medical Practices: O.A., B.E., Concept: O.A., B.E., Design: O.A., B.E., Data Collection or Processing: O.A., B.E., Analysis or Interpretation: O.A., B.E., Literature Search: O.A., B.E., Writing: O.A., B.E.

Conflict of Interest: No conflict of interest was declared by the authors.

Financial Disclosure: The authors declared that this study received no financial support.

\section{REFERENCES}

1. Smith CA, Gutmann L. Myotonic Dystrophy Type 1 Management and Therapeutics. Curr Treat Options Neurol 2016;18:52.

2. Thornton CA. Myotonic dystrophy. Neurol Clin 2014;32:705-19.

3. Mathieu J, Boivin H, Meunier D, Gaudreault M, Bégin P. Assessment of a disease-specific muscular impairment rating scale in myotonic dystrophy. Neurology 2001;56:336-40. 
4. Kersten HM, Roxburgh RH, Child N, Polkinghorne PJ, Frampton C, Danesh-Meyer HV. Epiretinal membrane: a treatable cause of visual disability in myotonic dystrophy type 1. J Neurol 2014;261:37-44.

5. Gemenetzi M, De Salvo G, Lotery AJ. Central serous chorioretinopathy: an update on pathogenesis and treatment. Eye (Lond) 2010;24:1743-56.

6. Yazgan S, Arpaci D, Celik HU, Dogan M, Isık I. Macular Choroidal Thickness May Be the Earliest Determiner to Detect the Onset of Diabetic Retinopathy in Patients with Prediabetes: A Prospective and Comparative Study. Curr Eye Res 2017;42:1039-47.

7. Bolukbasi S, Erden B, Cakir A, Bayat AH, Elcioglu MN, Yurttaser Ocak $S$, et al. Pachychoroid Pigment Epitheliopathy and Choroidal Thickness Changes in Coeliac Disease. J Ophthalmol 2019;2019:6924191.

8. Kurt MM, Akpolat C, Evliyaoglu F, Yilmaz M, Ordulu F. Evaluation of Retinal Neurodegeneration and Choroidal Thickness in Patients with Inactive Graves' Ophthalmopathy. Klin Monbl Augenheilkd 2021 Mar 17. English.

9. Choi MG, Chung H, Yoon YH, Kim JT. Effects of Systemic Profiles on Choroidal Thickness in Treatment-Naïve Eyes With Diabetic Retinopathy. Invest Ophthalmol Vis Sci 2020;61:12.

10. Okamoto M, Yamashita M, Ogata N. Effects of intravitreal injection of ranibizumab on choroidal structure and blood flow in eyes with diabetic macular edema. Graefes Arch Clin Exp Ophthalmol 2018;256:885-92.
11. Alsaggaf R, Pfeiffer RM, Wang Y, St George DMM, Zhan M, Wagner KR, et al. Diabetes, metformin and cancer risk in myotonic dystrophy type I. Int J Cancer 2020;147:785-92.

12. Ikeda KS, Iwabe-Marchese C, França MC Jr, Nucci A, Carvalho KM. Myotonic dystrophy type 1: frequency of ophthalmologic findings. Arq Neuropsiquiatr 2016;74:183-8.

13. Kimizuka Y, Kiyosawa M, Tamai M, Takase S. Retinal changes in myotonic dystrophy. Clinical and follow-up evaluation. Retina 1993;13:129-35.

14. Hayasaka S, Kiyosawa M, Katsumata S, Honda M, Takase S, Mizuno K. Ciliary and retinal changes in myotonic dystrophy. Arch Ophthalmol 1984;102:88-93.

15. Ng CH, Cheung N, Wang JJ, Islam AF, Kawasaki R, Meuer SM, et al. Prevalence and risk factors for epiretinal membranes in a multi-ethnic United States population. Ophthalmology 2011;118:694-9.

16. Koh V, Cheung CY, Wong WL, Cheung CM, Wang JJ, Mitchell P, et al. Prevalence and risk factors of epiretinal membrane in Asian Indians. Invest Ophthalmol Vis Sci 2012;53:1018-22.

17. Kim US, Kim JS, Hwang JM. A case of myotonic dystrophy with pigmentary retinal changes. Korean J Ophthalmol 2009;23:121-3.

18. Zinkernagel MS, Hornby SJ, MacLaren RE. Choroidal new vessels in type 1 myotonic dystrophy-related macular dystrophy respond to anti-VEGF therapy. Eye (Lond) 2012;26:1595-6. 